\title{
Positive solutions of singular beam equations with the bending term
}

\section{Xuemei Zhang ${ }^{1 *}$ and Meiqiang Feng ${ }^{2}$}

\footnotetext{
*Correspondence: zxm74@sina.com 1 Department of Mathematics and Physics, North China Electric Power University, Beijing, 102206, People's Republic of China

Full list of author information is available at the end of the article
}

\begin{abstract}
Using a new technique for dealing with the bending term of beam equations, we consider the existence and multiplicity of positive solutions for a beam equation. Besides achieving new results, upper and lower bounds for these positive solutions will also be provided. The results are shown by using a novel technique and fixed point theories.
\end{abstract}

Keywords: positive solution; singularity; beam equation with the bending term; fixed point theorem

\section{Introduction}

In this paper, we shall investigate the existence and multiplicity of positive solutions for the fourth order differential equation with integral boundary conditions

$$
\left\{\begin{array}{l}
y^{(4)}(t)=\omega(t) F\left(t, y(t), y^{\prime \prime}(t)\right), \quad 0<t<1 \\
y(0)=y(1)=\int_{0}^{1} h(s) y(s) d s \\
a y^{\prime \prime}(0)-b y^{\prime \prime \prime}(0)=\int_{0}^{1} g(s) y^{\prime \prime}(s) d s \\
a y^{\prime \prime}(1)+b y^{\prime \prime \prime}(1)=\int_{0}^{1} g(s) y^{\prime \prime}(s) d s
\end{array}\right.
$$

where $a, b>0, F:[0,1] \times R \times R \rightarrow R$ is continuous, and the $y^{\prime \prime}$ in $F$ is the bending moment term which represents bending effect. Problem (1.1) is often referred to as the deformation of an elastic beam under a variety of boundary conditions; for details, see [1-18]. Most research papers on beam equations consider nonlinear terms that $F$ in (1.1) involves $y$ only, and derivative-dependent nonlinearities are seldom tackled; see [3-7, 9-11] to name a few.

Some classical tools such as fixed point theorems in cones [5, 8-11, 17, 18], the method of lower and upper solutions $[8,16]$, and the monotone iterative method $[9,12]$ and the theory of critical point theory and variational methods $[6,13,14]$ have been widely used to study beam equations.

Some new techniques via appropriate transformation are proved to be very effective in studying the solvability of differential equations. Such techniques have attracted the attention of Zhang et al. [19] and Wong [20], etc. In [19], Zhang et al. considered the existence

(c) 2015 Zhang and Feng. This article is distributed under the terms of the Creative Commons Attribution 4.0 International License (http://creativecommons.org/licenses/by/4.0/), which permits unrestricted use, distribution, and reproduction in any medium, provided you give appropriate credit to the original author(s) and the source, provide a link to the Creative Commons license, and indicate if changes were made. 
of positive solutions of the following problems:

$$
\left\{\begin{array}{l}
x^{(n)}(t)+f\left(t, x(t), x^{\prime}(t), \ldots, x^{(n-2)}(t)\right)=\theta, \quad t \in J, t \neq t_{k}, k=1,2, \ldots, m \\
\left.\Delta x^{(n-1)}\right|_{t=t_{k}}=-I_{k}\left(x^{(n-2)}\left(t_{k}\right)\right), \quad k=1,2, \ldots, m \\
x^{(i)}(0)=\theta, \quad i=0,1, \ldots, n-3 \\
x^{(n-2)}(0)=x^{(n-2)}(1)=\int_{0}^{1} g(t) x^{(n-2)}(t) d t
\end{array}\right.
$$

where $J=[0,1]$.

Firstly, by means of the transformation

$$
x^{(n-2)}(t)=y(t)
$$

the authors converted (1.2) into

$$
\left\{\begin{array}{l}
x^{(n-2)}(t)=y(t), \quad t \in J \\
x^{(i)}(0)=\theta, \quad i=1,2, \ldots, n-3
\end{array}\right.
$$

and

$$
\left\{\begin{array}{l}
-y^{\prime \prime}(t)=f\left(t, x(t), x^{\prime}(t), \ldots, x^{(n-2)}(t)\right), \quad t \in J, t \neq t_{k}, \\
\left.\Delta y^{\prime}\right|_{t=t_{k}}=-I_{k}\left(y\left(t_{k}\right)\right), \quad k=1,2, \ldots, m, \\
y(0)=y(1)=\int_{0}^{1} g(t) y(t) d t
\end{array}\right.
$$

Then it follows from Lemma 2.3 in [19] that they converted the results obtained for problem (1.2) to the counterpart for problem (1.3).

In [20], Wong transformed the following problems:

$$
\left\{\begin{array}{l}
(-1)^{m} y^{(2 m+1)}(t)=F\left(t, y(t), y^{\prime}(t)\right), \quad 0<t<1, \\
y(0)=0, \quad y^{(2 k-1)}(0)=y^{(2 k-1)}(1)=0, \quad 0 \leq k \leq m,
\end{array}\right.
$$

into

$$
\left\{\begin{array}{l}
y^{\prime}(t)=x(t), \quad t \in J \\
y(0)=0
\end{array}\right.
$$

and

$$
\left\{\begin{array}{l}
(-1)^{m} x^{(2 m)}(t)=F\left(t, y(t), y^{\prime}(t)\right), \quad 0<t<1 \\
x^{(2 k-2)}(0)=x^{(2 k-2)}(1)=0, \quad 1 \leq k \leq m
\end{array}\right.
$$

by using $y^{\prime}(t)=x(t)$. So the existence of a solution of the complementary Lidstone boundary value problem (1.4) follows from the existence of a solution of the Lidstone boundary value problem (1.5). We notice that the above paper requires that $F$ satisfies some assumptions of monotonicity which are essential for the technique used.

Being directly inspired by $[19,20]$, in the present paper, by using transformation techniques and fixed point theories, the authors shall prove some new and more general results of the existence of at least one or two positive solutions for problem (1.1). The main features of this paper are as follows. Firstly, comparing with [1-18], besides achieving new results, 
estimates on the norms of these solutions will also be provided. Secondly, we transform problem (1.1) into a differential system without bending term, i.e., the technique to deal with bending term is completely different from that of $[8,16-18]$. Finally, it is pointed out that we do not need any monotone assumption on $F$, which is weaker than the corresponding assumptions on $F$ in [20].

On the other hand, boundary value problems with integral boundary conditions arise naturally in thermal conduction problems [21], semiconductor problems [22], hydrodynamic problems [23] and so on. It is interesting to point out that such problems include two, three, multi-point and nonlocal boundary value problems as special cases and have been extensively studied in the last ten years (see, for example, [24-30]). Therefore, it is important to study fourth order elasticity problems with integral boundary conditions.

The rest of the paper is organized as follows. In Section 2, we provide some preliminaries and lemmas. In particular, we transform problem (1.1) into a differential system without the bending moment term. In Section 3, the main results will be stated and proved.

\section{Preliminaries}

To establish the existence of positive solutions for problem (1.1), let us list the following assumptions, which will hold throughout this paper:

$\left(\mathrm{H}_{1}\right) \omega \in C((0,1),[0,+\infty))$ with $0<\int_{0}^{1} \omega(s) d s<\infty$ and $\omega$ does not vanish on any subinterval of $(0,1)$;

$\left(\mathrm{H}_{2}\right) \quad F \in C([0,1] \times[0,+\infty) \times(-\infty, 0],[0,+\infty))$;

$\left(\mathrm{H}_{3}\right) g, h \in L^{1}[0,1]$ are nonnegative and $\mu \in[0, a), v \in[0,1)$, where

$$
\mu=\int_{0}^{1} g(s) d s, \quad v=\int_{0}^{1} h(s) d s .
$$

Lemma 2.1 [11] Assume that $\left(\mathrm{H}_{3}\right)$ holds. Then, for any $x \in C[0,1]$, the boundary value problem

$$
\left\{\begin{array}{l}
-y^{\prime \prime}(t)=x(t), \quad 0<t<1 \\
y(0)=y(1)=\int_{0}^{1} h(s) y(s) d s
\end{array}\right.
$$

has a unique solution y given by

$$
y(t)=\int_{0}^{1} H_{1}(t, s) x(s) d s
$$

where

$$
\begin{aligned}
& H_{1}(t, s)=G(t, s)+\frac{1}{1-v} \int_{0}^{1} G_{1}(s, \tau) h(\tau) d \tau, \\
& G_{1}(t, s)= \begin{cases}t(1-s), & 0 \leq t \leq s \leq 1 \\
s(1-t), & 0 \leq s \leq t \leq 1 .\end{cases}
\end{aligned}
$$

Taking into account (2.2) and (2.3), problem (1.1) reduces to the following problem:

$$
\left\{\begin{array}{l}
x^{\prime \prime}(t)=-\omega(t) F\left(t, \int_{0}^{1} H_{1}(t, s) x(s) d s,-x(t)\right), \quad 0<t<1, \\
a x(0)-b x^{\prime}(0)=\int_{0}^{1} g(s) x(s) d s, \quad a x(1)+b x^{\prime}(1)=\int_{0}^{1} g(s) x(s) d s .
\end{array}\right.
$$


Lemma 2.2 [11] Assume that $\left(\mathrm{H}_{1}\right)-\left(\mathrm{H}_{3}\right)$ hold. Then boundary value problem (2.6) has a unique solution $x$ given by

$$
x(t)=\int_{0}^{1} H_{2}(t, s) \omega(s) F\left(s, \int_{0}^{1} H_{1}(s, \tau) x(\tau) d \tau,-x(s)\right) d s,
$$

where

$$
\begin{aligned}
& H_{2}(t, s)=G_{2}(t, s)+\frac{1}{a-\mu} \int_{0}^{1} G_{2}(s, \tau) g(\tau) d \tau, \\
& G_{2}(t, s)=\frac{1}{d} \begin{cases}(b+a s)(b+a(1-t)) & \text { if } 0 \leq s \leq t \leq 1, \\
(b+a t)(b+a(1-s)) & \text { if } 0 \leq t \leq s \leq 1,\end{cases} \\
& d=a(2 b+a) .
\end{aligned}
$$

If problem (2.6) has a solution $x^{*}$, then by (2.3) problem (1.1) has a solution given by

$$
y^{*}(t)=\int_{0}^{1} H_{1}(t, s) x^{*}(s) d s
$$

So the existence of a solution of problem (1.1) follows from the existence of a solution of problem (2.6).

Lemma 2.3 [11] For $t, s \in J$, we have the following results:

$$
\begin{aligned}
& G_{1}(t, t) G_{1}(s, s) \leq G_{1}(t, s) \leq G_{1}(s, s) \leq \frac{1}{4}, \\
& \frac{1}{d} b^{2} \leq G_{2}(t, s) \leq G_{2}(s, s) \leq \frac{1}{d}(b+a)^{2} .
\end{aligned}
$$

Lemma 2.4 [11] Let $\left(\mathrm{H}_{3}\right)$ hold. Then we obtain the following results:

$$
\begin{aligned}
& \rho G_{1}(s, s) \leq H_{1}(t, s) \leq \gamma G_{1}(s, s) \leq \frac{1}{4} \gamma, \quad \forall t, s \in J, \\
& \rho_{1} \leq H_{2}(t, s) \leq H_{2}(s, s) \leq \rho_{2}, \quad \forall t, s \in J
\end{aligned}
$$

where

$$
\begin{aligned}
& \gamma=\frac{1}{1-v}, \quad \rho=\frac{\int_{0}^{1} G(\tau, \tau) h(\tau) d \tau}{1-v}, \quad \rho_{1}=\frac{b^{2} \gamma_{1}}{a+2 b}, \\
& \rho_{2}=\frac{\gamma_{1}(b+a)^{2}}{a+2 b}, \quad \gamma_{1}=\frac{1}{a-\mu} .
\end{aligned}
$$

It is clear from (2.3) that $\left\|y^{*}\right\| \leq \frac{\gamma}{4}\left\|x^{*}\right\|$; moreover, if $x^{*}$ is positive, so is $y^{*}$.

Let $E=C[0,1]$. It is well known that $E$ is a real Banach space with the norm $\|\cdot\|$ defined by $\|x\|=\max _{t \in J}|x(t)|$.

Define an operator $T: E \rightarrow E$ as follows:

$$
(T x)(t)=\int_{0}^{1} H_{2}(t, s) \omega(s) F\left(s, \int_{0}^{1} H_{1}(s, \tau) x(\tau) d \tau,-x(s)\right) d s .
$$


Let $C$ be a cone in $E$ which is defined as

$$
C=\{x \in E: x \geq 0, x(t) \geq \delta\|x\|, t \in J\},
$$

where

$$
\delta=\frac{\rho_{1}}{\rho_{2}} .
$$

It is easy to see that $E$ is a closed convex cone of $E$.

Lemma 2.5 Let $\left(\mathrm{H}_{1}\right)-\left(\mathrm{H}_{3}\right)$ hold. Then we have $T(C) \subset C$, and $T: C \rightarrow C$ is completely continuous.

Proof In view of condition (2.13), we see that

$$
\begin{aligned}
\|T x\| & =\max _{t \in J} \int_{0}^{1} H_{2}(t, s) \omega(s) F\left(s, \int_{0}^{1} H_{1}(s, \tau) x(\tau) d \tau,-x(s)\right) d s \\
& \leq \rho_{2} \int_{0}^{1} \omega(s) F\left(s, \int_{0}^{1} H_{1}(s, \tau) x(\tau) d \tau,-x(s)\right) d s .
\end{aligned}
$$

Moreover, it follows from (2.13) and (2.14) that

$$
\begin{aligned}
(T x)(t) & =\int_{0}^{1} H_{2}(t, s) \omega(s) F\left(s, \int_{0}^{1} H_{1}(s, \tau) x(\tau) d \tau,-x(s)\right) d s \\
& \geq \rho_{1} \int_{0}^{1} \omega(s) F\left(s, \int_{0}^{1} H_{1}(s, \tau) x(\tau) d \tau,-x(s)\right) d s \\
& \geq \frac{\rho_{1}}{\rho_{2}} \rho_{2} \int_{0}^{1} \omega(s) F\left(s, \int_{0}^{1} H_{1}(s, \tau) x(\tau) d \tau,-x(s)\right) d s \\
& \geq \delta\|T x\|, \quad t \in J .
\end{aligned}
$$

This proves that $T(C) \subset C$.

Next, by standard methods and the Ascoli-Arzelà theorem, one can prove that $T: C \rightarrow$ $C$ is completely continuous.

To obtain positive solutions of problem (1.1), the following fixed point theorem in cones, which can be found in [31], p.94, is fundamental.

Lemma 2.6 Let $P$ be a cone in a real Banach space E. Assume $\Omega_{1}, \Omega_{2}$ are bounded open sets in $E$ with $0 \in \Omega_{1}, \bar{\Omega}_{1} \subset \Omega_{2}$. If

$$
A: P \cap\left(\bar{\Omega}_{2} \backslash \Omega_{1}\right) \rightarrow P
$$

is completely continuous such that either

(a) $\|A x\| \leq\|x\|, \forall x \in P \cap \partial \Omega_{1}$ and $\|A x\| \geq\|x\|, \forall x \in P \cap \partial \Omega_{2}$, or

(b) $\|A x\| \geq\|x\|, \forall x \in P \cap \partial \Omega_{1}$ and $\|A x\| \leq\|x\|, \forall x \in P \cap \partial \Omega_{2}$,

then $A$ has at least one fixed point in $P \cap\left(\bar{\Omega}_{2} \backslash \Omega_{1}\right)$. 


\section{Main results}

In this section, we apply Lemma 2.6 to establish the existence of positive solutions for problem (1.1). We begin by introducing the following conditions on $F(t, u, v)$ :

$\left(\mathrm{H}_{4}\right)$ There exist two positive constants $r, R$ with $\left(\frac{\gamma}{4}+1\right) r<(\sigma+\delta) R$ such that:

$$
\begin{aligned}
& F(t, u, v) \leq \frac{1}{\rho_{2} \eta} r, \quad \forall t \in J,|u|+|v| \leq\left(\frac{\gamma}{4}+1\right) r \\
& F(t, u, v) \geq \frac{1}{\rho_{1} \eta \delta} R, \quad \forall t \in J,|u|+|v| \geq(\sigma+\delta) R,
\end{aligned}
$$

where

$$
\eta=\int_{0}^{1} \omega(s) d s, \quad \sigma=\rho \delta \beta, \quad \beta=\int_{0}^{1} s(1-s) d s=\frac{1}{6} .
$$

Theorem 3.1 Assume that $\left(\mathrm{H}_{1}\right)-\left(\mathrm{H}_{4}\right)$ hold. Then we have the following conclusions:

(i) Problem (2.6) has (at least) one positive solution $x \in C$ such that

$$
\delta r \leq x(t) \leq \frac{1}{\delta} R, \quad t \in J .
$$

(ii) Problem (1.1) has (at least) one positive solution y such that

$$
\left\{\begin{array}{l}
y(t)=\int_{0}^{1} H_{1}(t, s) x(s) d s, \quad t \in J \\
\|y\| \leq \frac{\gamma}{4}\|x\| ; \\
y(t) \geq \sigma\|x\|, \quad t \in J
\end{array}\right.
$$

We further have

$$
\sigma \delta r \leq y(t) \leq \frac{\gamma}{4 \delta} R, \quad t \in J .
$$

Proof Let $T$ be the cone preserving, completely continuous operator that was defined by (2.14).

Let $x \in C$ with $\|x\|=r$. Then $0 \leq x(t) \leq r, t \in J$, and $0 \leq \int_{0}^{1} H_{1}(s, \tau) x(\tau) d \tau \leq \frac{\gamma}{4} r$. And hence, for $x \in C$ with $\|x\|=r$, we have

$$
\left|\int_{0}^{1} H_{1}(s, \tau) x(\tau) d \tau\right|+|-x(t)| \leq\left(\frac{\gamma}{4}+1\right) r
$$

Then it follows from (3.1) that

$$
\begin{aligned}
\|T x\| & =\max _{t \in J} \int_{0}^{1} H_{2}(t, s) \omega(s) F\left(s, \int_{0}^{1} H_{1}(s, \tau) x(\tau) d \tau,-x(s)\right) d s \\
& \leq \rho_{2} \eta \frac{1}{\rho_{2} \eta} r=r .
\end{aligned}
$$

Now if we let $\Omega_{1}=\{x \in C:\|x\|<r\}$, then (3.6) shows that

$$
\|T x\| \leq\|x\|, \quad x \in \partial \Omega_{1} .
$$


Further, let

$$
R_{1}=\frac{1}{\delta} R,
$$

and

$$
\Omega_{2}=\left\{x \in C:\|x\|<R_{1}\right\} .
$$

Then $x \in C$ and $\|x\|=R_{1}$ imply

$$
x(t) \geq \delta x(s), \quad t, s \in J,
$$

that is,

$$
x(t) \geq \delta R_{1}=R, \quad t \in J .
$$

Hence, $x(t) \geq R$ for all $t \in J$, and

$$
\int_{0}^{1} H_{1}(s, \tau) x(\tau) d \tau \geq \rho \int_{0}^{1} G(\tau, \tau) x(\tau) d \tau \geq \rho \beta \delta\|x\|=\sigma\|x\| .
$$

So

$$
\left|\int_{0}^{1} H_{1}(s, \tau) x(\tau) d \tau\right|+|-x(t)| \geq(\sigma+\delta) R .
$$

Using condition (3.2), it follows from $x \in C$ and $\|x\|=R_{1}$ that

$$
\|T x\|=\max _{t \in J} \int_{0}^{1} H_{2}(t, s) \omega(s) F\left(s, \int_{0}^{1} H_{1}(s, \tau) x(\tau) d \tau,-x(s)\right) d s \geq \rho_{1} \eta \frac{1}{\rho_{1} \eta \delta} R=\frac{R}{\delta}=R_{1},
$$

that is, $x \in \partial \Omega_{2}$ implies

$$
\|T x\| \geq\|x\| .
$$

It now follows from Lemma 2.6 that problem (2.6) has (at least) one positive solution $x \in \bar{\Omega}_{2} \backslash \Omega_{1}$ satisfying (3.3).

It is observed from (2.3) that problem (1.1) has (at least) one positive solution $y$ such that

$$
y=\int_{0}^{1} H_{1}(t, s) x_{1}(s) d s, \quad t \in J \text { and }\|y\| \leq \frac{\gamma}{4}\|x\| .
$$

Moreover, since $x \in C$, we get for $t \in J$

$$
\begin{aligned}
y(t) & =\int_{0}^{1} H_{1}(t, s) x(s) d s \\
& \geq \rho \int_{0}^{1} G(s, s) x(s) d s
\end{aligned}
$$




$$
\begin{aligned}
& \geq \rho \beta \delta\|x\| \\
& =\sigma\|x\| .
\end{aligned}
$$

Then we get (3.4).

Further, it follows from (3.3) and (3.4) that (3.5) holds.

In Theorem 3.2 we assume the following condition on $f(t, u, v)$ :

$\left(\mathrm{H}_{5}\right)$ There exist two positive constants $r, R$ with $\left(\frac{\gamma}{4}+1\right) r<R$ such that:

$$
\begin{aligned}
& F(t, u, v) \geq \frac{1}{\rho_{1} \eta(\sigma+\delta)}(|u|+|v|), \quad \forall t \in J,|u|+|v| \leq\left(\frac{\gamma}{4}+1\right) r \\
& F(t, u, v) \leq \frac{1}{2 \rho_{2} \eta\left(\frac{\gamma}{4}+1\right)}(|u|+|v|), \quad \forall t \in J,|u|+|v| \geq R
\end{aligned}
$$

and write

$$
M=\max _{\forall t \in J,|u|+|v| \leq R} F(t, u, v)
$$

Theorem 3.2 Assume that $\left(\mathrm{H}_{1}\right)-\left(\mathrm{H}_{3}\right)$ and $\left(\mathrm{H}_{5}\right)$ hold. Then we have the following conclusions:

(i) Problem (2.6) has (at least) one positive solution $x \in C$ such that

$$
\delta r \leq x(t) \leq \max \left\{2 R, 2 \rho_{2} \eta M\right\}, \quad t \in J .
$$

(ii) Problem (1.1) has (at least) one positive solution y such that (3.4) holds. We further have

$$
\sigma \delta r \leq y(t) \leq \max \left\{\frac{\gamma R}{4(\sigma+\delta)}, \frac{\rho_{2} \eta M \gamma}{2}\right\}, \quad t \in J
$$

Proof Let $x \in C$ with $\|x\|=r$. Then $0 \leq x(t) \leq r, t \in J$, and $0 \leq \int_{0}^{1} H_{1}(s, \tau) x(\tau) d \tau \leq \frac{\gamma}{4} r$. And hence for $x \in C$ with $\|x\|=r$, we have

$$
(\sigma+\delta) r \leq\left|\int_{0}^{1} H_{1}(s, \tau) x(\tau) d \tau\right|+|-x(t)| \leq\left(\frac{\gamma}{4}+1\right) r
$$

and it follows from condition $\left(\mathrm{H}_{5}\right)$ that

$$
\begin{aligned}
\|T x\| & =\max _{t \in J} \int_{0}^{1} H_{2}(t, s) \omega(s) F\left(s, \int_{0}^{1} H_{1}(s, \tau) x(\tau) d \tau,-x(s)\right) d s \\
& \geq \rho_{1} \eta \frac{1}{\rho_{1} \eta(\sigma+\delta)}(\sigma+\delta) r=r
\end{aligned}
$$

that is, $x \in \partial \Omega_{1}$ implies that

$$
\|T x\| \geq\|x\| .
$$


Next, we turn to (3.11) and (3.12). From (3.11) and (3.12), we have

$$
F(t, u, v) \leq M+\frac{1}{2 \rho_{2} \eta\left(\frac{\gamma}{4}+1\right)}(|u|+|v|), \quad(t, u, v) \in J \times[0, \infty) \times(-\infty, 0]
$$

Further, let

$$
R_{2}>\max \left\{2 \rho_{2} \eta M, \frac{R}{\sigma+\delta}\right\}
$$

and

$$
\Omega_{3}=\left\{x \in C:\|x\|<R_{2}\right\} .
$$

Notice that for $x \in \partial \Omega_{3}$ we have

$$
R<(\sigma+\delta) R_{2} \leq\left|\int_{0}^{1} H_{1}(s, \tau) x(\tau) d \tau\right|+|-x(t)| \leq\left(\frac{1}{4} \gamma+1\right) R_{2} .
$$

Thus, for $x \in \partial \Omega_{3}$, it follows from (3.17) that

$$
\begin{aligned}
\|T x\| & =\max _{t \in J} \int_{0}^{1} H_{2}(t, s) \omega(s) F\left(s, \int_{0}^{1} H_{1}(s, \tau) x(\tau) d \tau,-x(s)\right) d s \\
& \leq \rho_{2} \int_{0}^{1} \omega(s)\left(M+\frac{1}{2 \rho_{2} \eta} R_{2}\right) d s \\
& \leq \rho_{2} \eta\left(M+\frac{1}{2 \rho_{2} \eta} R_{2}\right) \\
& <R_{2},
\end{aligned}
$$

that is, $x \in \partial \Omega_{3}$ implies

$$
\|T x\|<\|x\| .
$$

It now follows from Lemma 2.6 that problem (2.6) has (at least) one positive solution $x \in \bar{\Omega}_{3} \backslash \Omega_{1}$ satisfying (3.13).

It follows from (2.3) that problem (1.1) has (at least) one positive solution $y$. Similar to the proof of (3.5), one can show that $y$ satisfies (3.14).

Theorem 3.3 Assume that $\left(\mathrm{H}_{1}\right)-\left(\mathrm{H}_{3}\right)$, (3.2) of $\left(\mathrm{H}_{4}\right)$ and (3.10) of $\left(\mathrm{H}_{5}\right)$ hold. In addition, letting $f$ satisfies the following condition:

$\left(\mathrm{H}_{6}\right)$ Let $l, \zeta$ and L satisfy

$$
0<l<\left(\frac{\gamma}{4}+1\right) l<\zeta<\left(\frac{\gamma}{4}+1\right) \zeta<\delta L<L
$$

If

$$
\max _{\forall t \in J, \zeta \leq|u|+|v| \leq\left(\frac{\gamma}{4}+1\right) \zeta} F(t, u, v)<\frac{1}{\rho_{2} \eta} \zeta
$$

then we have the following conclusions: 
(i) Problem (2.6) has (at least) two positive solutions $x_{1}, x_{2} \in C$ such that

$$
\delta l \leq x_{1}(t)<\zeta<\left(\frac{\gamma}{4}+1\right) \zeta<x_{2}(t) \leq L, \quad t \in J
$$

(ii) Problem (1.1) has (at least) two positive solutions $y_{1}, y_{2}$ such that for $i=1,2$,

$$
\left\{\begin{array}{l}
y_{i}(t)=\int_{0}^{1} H_{1}(t, s) x_{i}(s) d s, \quad t \in J \\
\left\|y_{i}\right\| \leq \frac{\gamma}{4}\left\|x_{i}\right\| \\
y_{i}(t) \geq \sigma\left\|x_{i}\right\|, \quad t \in J
\end{array}\right.
$$

We further have

$$
\left\{\begin{array}{l}
y_{1}(t)>\sigma \delta l, \quad t \in J \\
\left\|y_{2}\right\| \leq \frac{\gamma}{4} L
\end{array}\right.
$$

Proof If (3.10) of $\left(\mathrm{H}_{5}\right)$ holds, similar to the proof of (3.16), we can prove that

$$
\|T x\| \geq\|x\|, \quad x \in C,\|x\|=l
$$

If (3.2) of $\left(\mathrm{H}_{4}\right)$ holds, similar to the proof of (3.9), we have

$$
\|T x\| \geq\|x\|, \quad x \in C,\|x\|=L
$$

Finally, we show that

$$
\|T x\|<\|x\|, \quad x \in C,\|x\|=\zeta .
$$

In fact, for $x \in C$ with $\|x\|=\zeta$, we have

$$
x(t) \leq\|x\|=\zeta
$$

and

$$
\sigma \zeta \leq \int_{0}^{1} H_{1}(s, \tau) x(\tau) d \tau \leq \frac{\gamma}{4} \zeta
$$

Therefore,

$$
\zeta<(\sigma+1) \zeta \leq\left|\int_{0}^{1} H_{1}(s, \tau) x(\tau) d \tau\right|+|-x(s)| \leq\left(\frac{\gamma}{4}+1\right) \zeta,
$$

and hence it follows from $\left(\mathrm{H}_{6}\right)$ that

$$
\begin{aligned}
\|T x\| & =\max _{t \in J} \int_{0}^{1} H_{2}(t, s) \omega(s) F\left(s, \int_{0}^{1} H_{1}(s, \tau) x(\tau) d \tau,-x(s)\right) d s \\
& <\rho_{2} \eta \frac{1}{\rho_{2} \eta} \zeta=\zeta,
\end{aligned}
$$

which shows that (3.25) holds. 
Applying Lemma 2.6 to (3.23), (3.24) and (3.25) yields that problem (2.6) has (at least) two positive solutions $x_{1}, x_{2}$ with $x_{1} \in C_{\bar{l}, \zeta}=\{x \in C, l \leq\|x\|<\zeta\}, x_{2} \in C_{\zeta, \bar{L}}=\{x \in C$, $\left.\left(\frac{\gamma}{4}+1\right) \zeta<\|x\| \leq L\right\}$. Hence, since for $x_{1} \in C$ we have $x_{1}(t) \geq \delta\left\|x_{1}\right\|, t \in J$, it follows that (3.20) holds.

Similar to the proof of (3.4) and (3.5), one can show that (3.21) and (3.22) hold.

Remark 3.1 In Theorems 3.1-3.3, we generalize the results of $[8,16-18]$ in three main directions as follows:

(1) Upper and lower bounds for these positive solutions are given.

(2) Estimates on the norms of positive solutions are considered.

(3) The method to deal with the bending term of beam equations in this paper is completely different from that of $[8,16-18]$, which opens a new technique to study the beam equations with the bending term.

\section{Competing interests}

The authors declare that they have no competing interests.

\section{Authors' contributions}

XZ completed the main study and carried out the results of this article. MF checked the proofs and verified the calculation. All the authors read and approved the final manuscript.

\section{Author details}

'Department of Mathematics and Physics, North China Electric Power University, Beijing, 102206, People's Republic of China. ${ }^{2}$ School of Applied Science, Beijing Information Science \& Technology University, Beijing, 100192, People's Republic of China.

\section{Acknowledgements}

This work is sponsored by the project NSFC (11301178), the Fundamental Research Funds for the Central Universities (2014ZZD10, 2014MS58) and the Scientific Research Common Program of Beijing Municipal Commission of Education (KM201511232018). The authors are grateful to anonymous referees for their constructive comments and suggestions, which has greatly improved this paper.

Received: 25 November 2014 Accepted: 12 May 2015 Published online: 26 May 2015

\section{References}

1. Gupta, CP: Existence and uniqueness theorems for a bending of an elastic beam equation. Appl. Anal. 26, 289-304 (1988)

2. Agarwal, RP: Boundary Value Problems for Higher Order Differential Equations. World Scientific, Singapore (1986)

3. Liu, LS, Zhang, XG, Wu, YH: Positive solutions of fourth-order nonlinear singular Sturm-Liouville eigenvalue problems. J. Math. Anal. Appl. 326, 1212-1224 (2007)

4. Graef, JR, Qian, C, Yang, B: A three point boundary value problem for nonlinear fourth order differential equations. J. Math. Anal. Appl. 287, 217-233 (2003)

5. Anderson, DR, Avery, Rl: A fourth-order four-point right focal boundary value problem. Rocky Mt. J. Math. 36(2), 367-380 (2006)

6. Ma, TF: Positive solutions for a beam equation on a nonlinear elastic foundation. Math. Comput. Model. 39, 1195-1201 (2004)

7. Yao, QL: Positive solutions of a nonlinear elastic beam equation rigidly fastened on the left and simply supported on the right. Nonlinear Anal. 69, 1570-1580 (2008)

8. Bai, ZB: The method of lower and upper solutions for a bending of an elastic beam equation. J. Math. Anal. Appl. 248, 95-202 (2000)

9. Zhang, XG, Liu, LS: Positive solutions of fourth-order four-point boundary value problems with $p$-Laplacian operator. J. Math. Anal. Appl. 336, 1414-1423 (2007)

10. Zhang, XM, Feng, MQ, Ge, WG: Existence results for nonlinear boundary-value problems with integral boundary conditions in Banach spaces. Nonlinear Anal. 69, 3310-3321 (2008)

11. Zhang, XM, Ge, WG: Symmetric positive solutions of boundary value problems with integral boundary conditions. Appl. Math. Comput. 219, 3553-3564 (2012)

12. Sun, J, Wang, X: Monotone positive solutions for an elastic beam equation with nonlinear boundary conditions. Math. Probl. Eng. (2011). doi:10.1155/2011/609189

13. Yang, $L, C$ hen, $H$, Yang, $X$ : The multiplicity of solutions for fourth-order equations generated from a boundary condition. Appl. Math. Lett. 24, 1599-1603 (2011)

14. Cabada, A, Tersian, S: Multiplicity of solutions of a two point boundary value problem for a fourth-order equation. Appl. Math. Comput. 219, 5261-5267 (2013)

15. Han, G, Xu, Z: Multiple solutions of some nonlinear fourth-order beam equations. Nonlinear Anal. 68, 3646-3656 (2008) 
16. Ma, R, Zhang, J, Fu, S: The method of lower and upper solutions for fourth-order two-point boundary value problems. J. Math. Anal. Appl. 215, 415-422 (1997)

17. Li, Y: On the existence of positive solutions for the bending elastic beam equations. Appl. Math. Comput. 189 821-827 (2007)

18. Zhang, XM, Ge, WG: Positive solutions for a class of boundary-value problems with integral boundary conditions. Comput. Math. Appl. 58, 203-215 (2009)

19. Zhang, XM, Yang, XZ, Ge, WG: Positive solutions of $n$ th-order impulsive boundary value problems with integral boundary conditions in Banach spaces. Nonlinear Anal. 71, 5930-5945 (2009)

20. Wong, PJY: Triple solutions of complementary Lidstone boundary value problems via fixed point theorems. Bound. Value Probl. 2014, 125 (2014)

21. Cannon, JR: The solution of the heat equation subject to the specification of energy. Q. Appl. Math. 21, 155-160 (1963)

22. Ionkin, NI: Solution of a boundary value problem in heat conduction theory with nonlocal boundary conditions. Differ. Equ. 13, 294-304 (1977)

23. Chegis, RY: Numerical solution of a heat conduction problem with an integral boundary condition. Liet. Mat. Rink. 24, 209-215 (1984)

24. Boucherif, A: Second-order boundary value problems with integral boundary conditions. Nonlinear Anal. 70, 364-371 (2009)

25. Wang, YQ, Liu, LS, Wu, YH: Positive solutions for a nonlocal fractional differential equation. Nonlinear Anal. 74, 3599-3605 (2011)

26. Ahmad, B, Alsaedi, A, Alghamdi, BS: Analytic approximation of solutions of the forced Duffing equation with integral boundary conditions. Nonlinear Anal., Real World Appl. 9, 1727-1740 (2008)

27. Webb, JRL: Positive solutions of some three point boundary value problems via fixed point index theory. Nonlinear Anal. 47, 4319-4332 (2001)

28. Jiang, JQ, Liu, LS, Wu, YH: Second-order nonlinear singular Sturm-Liouville problems with integral boundary conditions. Appl. Math. Comput. 215, 1573-1582 (2009)

29. Liu, LS, Hao, XA, Wu, YH: Positive solutions for singular second order differential equations with integral boundary conditions. Math. Comput. Model. 57, 836-847 (2013)

30. Liu, LS, Liu, BM, Wu, YH: Nontrivial solutions for higher-order $m$-point boundary value problem with a sign-changing nonlinear term. Appl. Math. Comput. 217, 3792-3800 (2010)

31. Guo, DJ, Lakshmikantham, V: Nonlinear Problems in Abstract Cones. Academic Press, New York (1988)

\section{Submit your manuscript to a SpringerOpen ${ }^{\ominus}$ journal and benefit from:}

- Convenient online submission

Rigorous peer review

- Immediate publication on acceptance

- Open access: articles freely available online

- High visibility within the field

- Retaining the copyright to your article 